\title{
Qualität und Qualitätsmessung in der Pflege - \\ Theoretische Grundlagen und methodische Zugänge
}

\author{
Peter Hensen \\ (c) Der/die Autor(en) 2018 \\ K. Jacobs et al. (Hrsg.), Pflege-Report 2018 \\ https://doi.org/10.1007/978-3-662-56822-4_1
}

\section{Zusammenfassung}

Dieser Beitrag gibt eine Einführung in das Thema Qualität und Qualitätsmessung in der pflegerischen Versorgung. Hierzu wird zunächst eine modellhafte Annäherung an den Qualitätsbegriff vorgenommen sowie grundlegende handlungstheoretische Zugänge eröffnet. Darauf aufbauend werden theoretische und methodische Voraussetzungen der Qualitätsmessung vorgestellt, die Bestandteil jeder Auseinandersetzung mit dem Qualitätsbegriff sind. Qualitätsmessung gibt Auskunft über die Beschaffenheit des Leistungs- und Versorgungsgeschehens (deskriptiver Ansatz), aber auch darüber, ob und inwieweit die an die Versorgung gestellten Qualitätsanforderungen erfüllt werden (evaluativer Ansatz). Sie bedarf jedoch stets einer wissenschaftlichen Fundierung und sollte auf einem gleichermaßen professionell akzeptierten wie legitimierten Qualitätsverständnis aufbauen.

This chapter introduces the concept and measurement of quality in nursing care. First, definitions and meanings of quality in health and nursing care as well as action-theoretical approaches are described by a theory-driven approach. Subsequently, the paper deals with principal theoretical and methodological aspects of quality measurement which are necessary to discuss the numerous approaches of defining quality. In principle, measurement of quality provides data and information about the nature of health care services (descriptive approach), and whether and to what extent predefined quality requirements concerning health and nursing care are met (evaluative approach). However, quality measures always must be science-based and wellfounded and, in the same manner, they must be based on a predefined concept of quality which is accepted and legitimised by the health and nursing care professionals.

\section{$1.1 \quad$ Einleitung}

Die Qualität der pflegerischen Versorgung hat nicht nur breite öffentliche Aufmerksamkeit; sie unterliegt auch starker politischer Einflussnahme und Kontrolle (Geraedts et al. 2011). Das Qualitätsthema ist an vielen Stellen in der Sozialgesetzgebung zur Pflegeversicherung fest verankert, beispiels- weise als Element strukturgebender Mindestanforderungen (z. B. Maßstäbe und Grundsätze für die Qualität, Qualitätssicherung und Qualitätsdarstellung nach $₫ 113$ SGB XI), als Element der Leistungsüberprüfung im Sinne einer administrativ-kontrollierenden Qualitätssicherung (z. B. Qualitätsprüfungen nach $\$ 114$ SGB XI) oder als Element einer ver- bis geordneten Entwicklung 
fachlicher Leistungsstandards (z. B. Expertenstandards nach $₫ 113$ a SGB XI).

Im fachwissenschaftlichen Kontext der Gesundheits- und Pflegeberufe steht Qualität symbolhaft für eine »Gute Praxis« beruflichen bzw. professionellen Handelns; ein Handeln, dessen Ziel- und Zweckbestimmung gleichsam wie der dazugehörige methodische Handlungsraum durch die Berufsangehörigen vor- und mitbestimmt werden. Eine solche berufsund professionsbezogene Eigenständigkeit ist gleichzeitig immer auch eingebunden in einen institutionellen Leistungsrahmen, der von gesellschaftlichen und politischen Ansprüchen ebenso getragen wird wie von einrichtungsinternen bzw. einzelbetrieblichen Anforderungen und Erfordernissen einer sachgerechten Mittelerschließung und -verwendung.

In die Versorgungspraxis ist das Qualitätsthema vielfältig integriert. Konzepte traditioneller Qualitätssicherung, systematischer Qualitätsverbesserung oder professionsgeleiteter Qualitätsentwicklung durchdringen ganz selbstverständlich das Leistungsgeschehen wie auch das Selbstverständnis der Gesundheits- und Pflegeberufe; in den letzten Jahren haben Verfahren der vergleichenden Qualitätsmessung und -darstellung zunehmend Raum gewonnen. In diesem Beitrag soll eine kurze Einführung zu den wichtigen Begriffen und Konzepten der Qualitätsbestimmung und -gestaltung wie auch zu den methodischen Grundlagen der Qualitätsmessung und -beurteilung im Kontext der pflegerischen Versorgung gegeben werden.

\subsection{Annäherung an den Qualitätsbegriff}

Qualität ist bekanntermaßen keine Beobachtungsgröße, die allgemeingültig, absolut und vorhersehbar in Erscheinung tritt. Sie ist vielmehr ein Konstrukt, das sich unter dem Einfluss verschiedener Anspruchsgruppen, sachlicher Erfordernisse und individueller Erwartungen unterschiedlich formt ${ }^{1}$.

1 Technisch-funktional lässt sich eine deskriptiv-analytische Begriffsebene im Sinne der wertneutralen Beschreibung von Merkmalen und Eigenschaften (Beschaffenheitsbegriff) von einer normativ-evaluativen Begriffsebene im Sinne der Bildung von Wertmaßstäben und Werturteilen (Gütebegriff) unterscheiden.
Qualität ist hiernach ein normatives Konzept, das außerhalb von gesellschaftlichen und persönlichen Wertmaßstäben nicht denkbar wäre (Merchel 2013, S. 41). Innerhalb des Leistungsgeschehens materialisiert sich Qualität als ein pragmatisches Konzept von unterscheidbaren Anforderungen (»Sollen«), vorhandenen und einsetzbaren Fähigkeiten (»Können«) und einer dem Einzelfall angemessenen Vorstellung und Bereitschaft, wie und in welchem Ausmaß diesen Anforderungen zu entsprechen ist (»Wollen«).

Eine in dieser Weise zum Ausdruck gebrachte Relativität des Qualitätsbegriffs findet ihren sprachlichen Ausdruck häufig auch im Begriff einer kontextbezogenen bzw. »optimalen" Qualität, die sich von einer »maximalen« Qualität, d. h. von einem idealisierten Qualitätsverständnis, unterscheidet (Harteloh 2003). Für die Bezeichnung der Qualität sozialer und gesundheitlicher, sog. personenbezogener Dienstleistungen, wird häufig der Begriff Versorgungsqualität ${ }^{2}$ (je nach Versorgungsbereich und Fokussierung auch: Betreuungs-, Behandlungs- oder Pflegequalität) herangezogen; auch wenn damit unterschiedliche Inhalte und Zielsetzungen transportiert werden (Prütz 2012).

\subsubsection{Modelltheoretische Zugänge zum Qualitätsbegriff}

Um ein Konstrukt wie das der Versorgungsqualität auf der Ebene der Leistungserbringung fassen und letztendlich auch messen zu wollen, muss theoretische Vorarbeit geleistet werden. Bezugsgrößen hierfür liefern Qualitätsanforderungen, d. h. Aussagen über die erwartete oder erforderliche Beschaffenheit der jeweils zu betrachtenden Leistungseinheit, die in einem Qualitätsmodell operationalisiert werden. In den allermeisten Fällen machen Qualitätsmodelle überwiegend inhaltstheoretische Aussagen zu grundsätzlichen und wünschenswerten An-

2 Der Begriff »Versorgungsqualität« adressiert unterschiedliche Gestaltungsebenen; seine Verwendung betont jedoch stets die Bedeutung eines zugrundeliegenden professionellen Qualitätsverständnisses: „Quality of care is the degree to which health services for individuals and populations increase the likelihood of desired health outcomes and are consistent with current professional knowledge." (IOM 1990, S. 128f.) 
forderungen an die Qualität der Versorgung bzw. Pflege und führen diese listenartig als Kriterien oder Qualitätsdimensionen auf. In der Literatur werden hierzu oft genannt (Donabedian 1988; 1990; Maxwell 1992; Campbell et al. 2000; IOM 2001; Evans et al. 2001; Arah et al. 2006; Allen-Duck et al. 2017): Wirksamkeit (Efficacy, Effectiveness), Wirtschaftlichkeit (Efficiency, Economy), Zugang zur Versorgung (Accessibility, Access to care), Sicherheit (Safety), Gleichheit (Equity, Fairness), Angemessenheit (Appropriateness, Relevance to Need), Patientenorientierung (Social Acceptibility, PatientCenteredness, Responsiveness), Zufriedenheit (Satisfaction), Kontinuität (Continuity of Care, Integrated Care) oder Rechtzeitigkeit (Timeliness). Hierzu ergänzend und vertiefend werden für die Langzeitpflege häufig Aspekte der beziehungsorientierten Interaktion besonders betont (Rantz et al. 1998; 1999).

Wenngleich prozesstheoretische Aspekte über Wirkzusammenhänge oder prognostische Aussagen über die tatsächlich zu erreichende Qualität weitgehend unberücksichtigt bleiben, liefern Qualitätsaussagen dieser Art den wissenschaftlich geleiteten Referenzrahmen für ein Qualitätsverständnis, das von möglichst allen an der Pflege bzw. Versorgung Beteiligten geteilt wird. Derart multidimensionale Qualitätsaussagen haben gleichsam eine "Leitplankenfunktion « für die im Einzelnen vorzunehmende Spezifizierung der Qualitätsbestimmung im regionalen, lokalen und »Vor-Ort«-Versorgungsumfeld (Reerink 1990).

Als Strukturierungshilfe für die Qualitätsbestimmung im Gesundheitswesen wird üblicherweise die sequenzielle Gliederungssystematik nach Donabedian herangezogen, mit der die Leistungserbringung (personenbezogene Dienstleistung) anhand ihrer Struktur-, Prozess- und Ergebnismerkmale aufgefächert wird (Donabedian 1966; 1980, S. 81 ff.). Diese Trichotomie des Qualitätsbegriffs bietet zwar keine Definition von Qualität an sich; wohl aber eine brauchbare Operationalisierung:

- Strukturqualität (Structure): Sie betrachtet die strukturellen Voraussetzungen, die für die pflegerische Versorgung notwendig sind. Hierunter werden sämtliche personenbezogenen Voraussetzungen (z. B. Qualifikation, Fähig- keiten des Personals), materielle Elemente (z. B. bauliche, räumliche und apparative Ausstattung), aber auch organisatorische Elemente (z. B. Aufbauorganisation) der jeweils zu betrachtenden Leistungseinheit gefasst (»Über die richtigen Voraussetzungen verfügen «).

- Prozessqualität (Process): Sie betrachtet alle Aktivitäten, Tätigkeiten und Handlungen der versorgungsrelevanten Leistung (z. B. pflegerische Versorgung), d. h. die dazugehörigen Teilprozesse (z. B. Pflegeplanung, Pflegedokumentation, Pflegevisite) und Unterstützungsprozesse (z. B. Beschaffung, Reinigung). Prozessqualität bezieht sich auf die Art und Weise der Leistungserbringung (z. B. Ablauforganisation) hinsichtlich ihrer zeitlichen und sachlichen Erforderlichkeit inklusive der Einhaltung von Vorgaben und Beachtung von Standards (»Das Richtige richtig tun«).

- Ergebnisqualität (Outcome): Sie betrachtet die Resultate hinsichtlich ihrer (pflegerischen) Zielerreichung (z. B. Veränderung des Gesundheitszustands). Ergebnisqualität bezieht sich auf Versorgungsendpunkte (z. B. Lebensqualität, Teilhabe) oder auf sog. Surrogatparameter (z. B. Verbesserung einer Körperfunktion, Vermeidung von Schäden). Sie kann objektivierbare Veränderungen (z. B. Mobilitätsverbesserung) oder subjektive Bewertungen (z. B. Zufriedenheit) umfassen (»Den angestrebten, erreichbaren Zustand erreichen«).

Diese Art der Konzeptualisierung (Three-PartApproach) liefert eine bis heute gebräuchliche Referenzfolie für die dienstleistungsspezifische Produktionstheorie (Meyer und Mattmüller 1987; Fließ et al. 2005, S. 397), in der sich Dienstleistungen durch das Zusammentreten von Potenzialität, Performanz und Produkt konstituieren (vgl. Kleinaltenkamp 2001, S. 40). Diese Gliederungssystematik betrachtet explizit jedoch nicht die verschiedenen Akteure mit ihren unterscheidbaren Anforderungen und Handlungslogiken, insbesondere nicht die Sichtweise der Leistungsempfänger (Adressaten), sodass eine Anforderungsebene ergänzend zur Leistungsebene eingezogen werden muss (• Abb. 1.1).

Mit der Unterscheidung von »kundenbezogener Qualität«, "professionsbezogener Qualität« und 


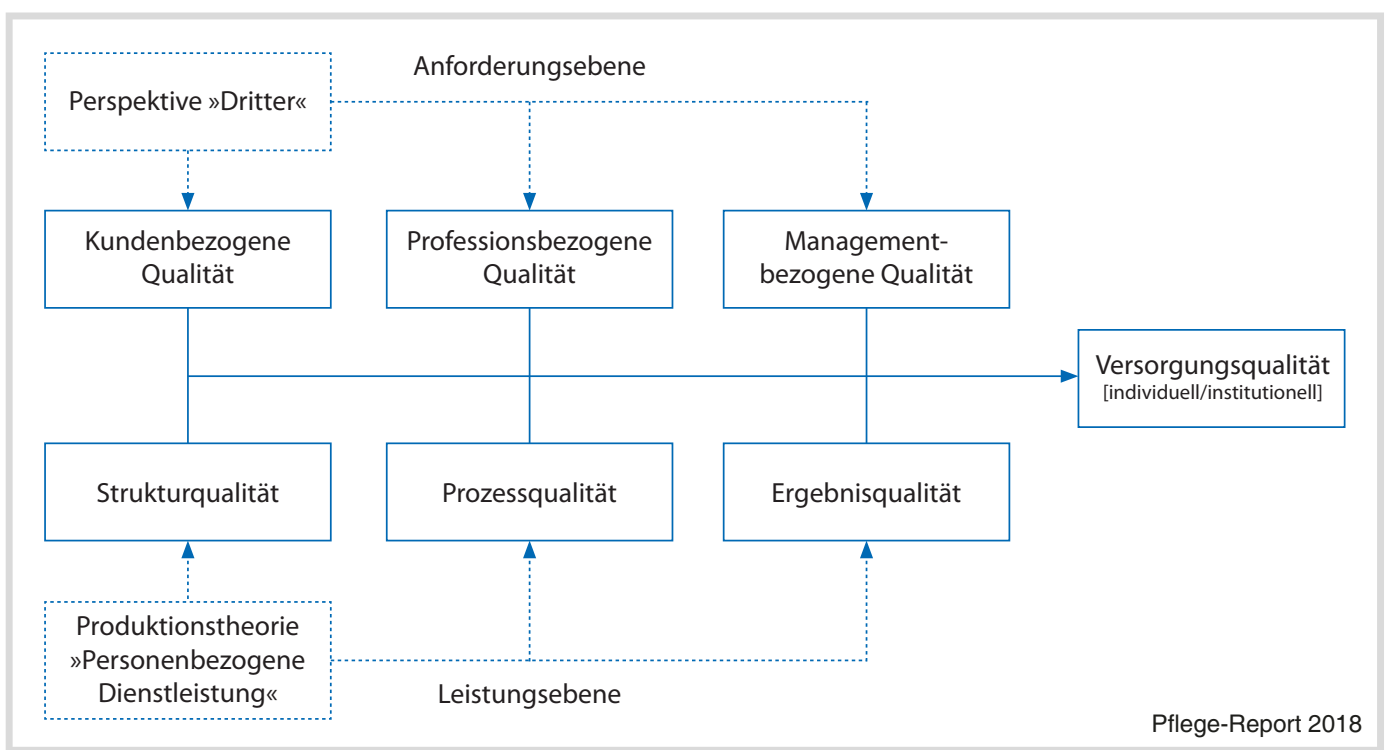

- Abb. 1.1 Gliederungssystematik der Qualitätsbestimmung

"managementbezogener Qualität« werden zusätzlich drei idealtypische Perspektiven bzw. Anspruchsgruppen voneinander differenziert (Øvretveit 1992; Piligrimiene und Buciuniene 2008), mit denen die Anforderungen an das Leistungsgeschehen gegliedert und konkretisiert werden können (Attree 1996). Für jedes Element der sequentiellen Gliederungssystematik lassen sich so spezifische Erfordernisse und Erwartungen an die Leistungserbringung formulieren, deren Erfüllung dann im Rahmen der Qualitätsmessung mit geeigneten Verfahren und Instrumenten geprüft wird.

Die kundenbezogene Qualität zielt auf die Erfüllung der von den Leistungsempfängern und Adressaten der Leistungsangebote (z. B. Nutzer, Bewohner, Klienten) gestellten Wünsche und Erwartungen an die Leistungserbringung. Es sind dies Anforderungen, die situations- und interaktionsübergreifend an die Leistungsangebote oder individuell an die konkrete Leistungserbringung gestellt werden. Daneben entspricht die professionsbezogene Qualität den Anforderungen bzw. den Vorstellungen und Möglichkeiten, die von den Angehörigen der Gesundheitsberufe bzw. Professionen an die Leistungserstellung gerichtet werden. Sie umfasst Festlegungen darüber, welche Leistungs- und Versorgungsangebote den Bedürfnissen und dem
Bedarf der Nutzer entsprechen, ebenso wie die richtigen Vorgehensweisen und Mittel ausgewählt und angewendet werden können, um im Einzelfall den Bedarfen, Wünschen und Erwartungen der Nutzer gerecht zu werden. Grundlage hierfür liefert ein in der Regel kodifiziertes und explizites Professionswissen (z. B. Studiendaten, Expertenstandards) wie auch ein informelles und implizit-praktisches Handlungswissen. Die managementbezogene Qualität entspricht den Anforderungen an die Bereitstellung und Organisation der Ressourcen und Verwendungsmittel, die innerhalb des institutionellen Leistungsrahmens notwendig sind, um sowohl den individuellen Wünschen und Erwartungen als auch den Bedarfen und versorgungsrelevanten Erforderlichkeiten entsprechen zu können. Diese Anforderungsperspektive zielt auf einen möglichst produktiven und effizienten Mitteleinsatz unter Beachtung der Erfordernisse übergeordneter Stellen (z. B. gesetzliche Festlegungen, behördliche Auflagen) oder anderer gesellschaftlicher (indirekter) Anspruchsgruppen (z. B. Spezifikation des Versorgungsauftrags). Sämtliche übergeordneten Anforderungen (»Perspektive Dritter«) wirken hiernach nicht direkt, sondern moduliert über die drei genannten Perspektiven auf die Leistungserstellungsebene (Hensen 2018, S. 29ff). 


\subsubsection{Handlungstheoretische Zugänge zur Qualitätsgestaltung}

Handlungstheoretisch lassen sich zwei methodische Grundhaltungen identifizieren (Verbeck 1998; Ruckstuhl et al. 2001), die in ihrer praktischen Anwendung untrennbar miteinander verbunden sind. Bei der ersten geht es grundsätzlich darum, festgelegten, allgemein gültigen oder vereinbarten Qualitätsanforderungen zu entsprechen (»Erfüllungsparadigma« der Qualitätssicherung ${ }^{3}$ ). Dahinter stehen Aktivitäten, die - als retrospektive Qualitätssicherung bezeichnet - im Rahmen des Leistungsgeschehens eine prüfende Funktion erfüllen und Anpassungen »im laufenden Betrieb« ermöglichen (Qualität durch Kontrolle und Korrektur); wie auch Maßnahmen, die - als vorbeugende Qualitätssicherung bezeichnet - präventive Maßnahmen der Fehlervermeidung und vorausschauende Maßnahmen zur Einhaltung definierter Qualitätsstandards bereithalten (Qualität durch Standardisierung und Korrektur- bzw. Vorbeugungsmaßnahmen). Aktivitäten dieser Art sind vielfach bereits Bestandteil eines professionellen oder kundenorientierten Selbstverständnisses, ohne dass sie zwangsläufig mit einem besonders zu benennenden Qualitätskonzept in Verbindung gebracht werden (z. B. hygienische Händedesinfektion, Pflegevisite). Begrifflich wird von interner Qualitätssicherung gesprochen, wenn das handlungsleitende Qualitätsverständnis und die daraus abgeleiteten Maßnahmen und Aktivitäten Gültigkeit für einen definierten Versorgungsbereich (z. B. Pflegeeinrichtung) und dessen Wirkradius besitzen. Eine externe Qualitätssicherung orientiert sich dagegen an Kriterien und Festlegungen, die von legitimierten Institutionen außerhalb eines solchen Versorgungsbereichs vorgegeben werden und ggf. auch von diesen ge-

3 Der Begriff "Qualitätssicherung« steht sowohl für die ursprünglich rein produkt- und herstellungsorientierte Darlegung einer vorliegenden Qualitätsfähigkeit (»Quality Assurance" im Sinne einer vertrauensbildenden "Zusicherung«). Er steht als begriffliche Metapher oft auch für die Gesamtheit aller qualitätswirksamen Aktivitäten und Zielsetzungen, deren Bereitstellung und Aufrechterhaltung ebenso wie ihr planvolles und systematisches Zusammenwirken in institutionellen Kontexten mit dem Begriff des Qualitätsmanagements belegt ist. prüft und überwacht werden können, sodass Vergleiche mit anderen Versorgungsbereichen möglich werden.

Die zweite Grundhaltung zielt auf Veränderungen von Zuständen, Voraussetzungen und Bedingungen mit dem Ziel, die messbare Qualität der Leistungen auf ein höheres Niveau zu heben (»Optimierungsparadigma« der Qualitätsverbesserung). Eine solche Qualitätsverbesserung orientiert sich in einem eng gefassten Verständnis an der Optimierung von vorhandenen Ressourcen, Vorgehensweisen und Mitteln (z. B. Personaleinsatzpläne, professionelle Praxisstandards). In einem weiter gefassten Verständnis umfasst sie alle Aktivitäten und Maßnahmen zur Erhöhung der Effektivität und Effizienz des institutionellen Leistungsrahmens, mit der ein zusätzlicher Nutzen sowohl für die Organisation als auch für ihre Nutzer erreicht werden soll (z. B. Audittätigkeiten, Verwirklichen von Mess- und Analyseverfahren). Werden Veränderungen nicht nur anlassbezogen und problemorientiert in Form von Einzelmaßnahmen realisiert, sondern planvoll und regelmäßig auf Grundlage einer qualitätsbezogenen Haltung durchgeführt, wird von ständiger oder kontinuierlicher Qualitätsverbesserung gesprochen.

Als integrierende Klammer für diese handlungsorientierten Grundhaltungen hat sich der vergleichsweise junge Begriff des Qualitätsmanagements etabliert, der im Sinne eines »leitenden und lenkenden «Gestaltungsansatzes (»Management von Qualität «) die vorgenannten Prinzipien miteinander vereint. Qualitätsmanagement bringt in einem institutionellen Kontext - vor allem in der Konnotation eines Umfassenden Qualitätsmanagements (auch: Total Quality Management, TQM) - die unternehmerische bzw. einrichtungsinterne Perspektive der Qualitätsgestaltung zum Ausdruck und versteht Qualität als eine organisationsweite Führungs- und Unternehmensstrategie (Dotchin und Oakland 1992; Zink und Schildknecht 1994). Diesen konzepthaft gefassten Gestaltungsansätzen können je nach Nomenklatur und Taxonomie eine Vielzahl von Unter- bzw. Querschnittsaufgaben (z. B. Qualitätsmessung, Qualitätsbewertung) zu- und untergeordnet werden.

Als ein gemeinsames Merkmal weisen die hier genannten »Wirkkonzepte der Qualitätsgestal- 
tung" (Hensen 2016, S. 49) einen hohen Systematisierungsgrad auf, der sich durch analytische (die Ausgangslage untersuchende) und intentionale (ziel- und zwecksetzende) Elemente bei der Realisierung qualitätsrelevanter Anforderungen auszeichnet. Dieser orientiert sich durchgängig am Prinzip der methodisch geleiteten Bemessung, Bewertung und Beurteilung (»Evaluationsparadigma« der Qualitätsgestaltung) entweder im Sinne der Überwachung des unmittelbaren Leistungsgeschehens (operative Evaluation) oder im Sinne der Erfolgskontrolle von Plan- und Zielgrößen (strategische Evaluation). Die Zusammenhänge von Analyse und Zielplanung, Ausführung und Überwachung sowie Messung und Beurteilung des Geschehens bzw. der Resultate treten funktional als Kreislaufmodell in Erscheinung und werden im Qualitätswesen modellhaft als PDCA-Zyklus (»Plan-DoCheck-Act») gefasst (Deming 1982). Konzeptübergreifend verfolgt eine solche Systematisierung stets die gleichen Absichten: das Definieren von Inhalten, Zielen und Teilzielen (für die Versorgung), das Planen und Verwirklichen von DurchführungsÜberwachungs-, Mess-, Analyse- und Verbesserungsmaßnahmen, das Überprüfen und Bewerten von Daten und Resultaten sowie das Treffen der richtigen Schlussfolgerungen und Ableiten von Konsequenzen.

\subsection{Grundlagen der Qualitäts- messung und -beurteilung}

Qualitätsmessung ist nicht einheitlich definiert. In einem weit gefassten Verständnis bezeichnet sie das Sammeln, Aufzeichnen und Verarbeiten von qualitätsrelevanten Daten sowie das gezielte Analysieren und Verdichten dieser Daten zu verwertbaren Informationen. Messen in einem sehr engen Begriffsverständnis bedeutet, den Betrag oder den Umfang eines Sachverhalts - im engeren Sinne eines Qualitätsmerkmals - in reproduzierbarer Weise und aus einer möglichst neutralen Position heraus zu ermitteln.

Die Qualitätsbeurteilung geht über ein solches Ermitteln von Merkmalsausprägungen und Sachverhalten hinaus. Sie ist immer kontextbezogen und interessengeleitet, $d$. h. sie hängt davon $a b$, welche
Messverfahren ausgewählt und angewendet werden, welche Normen, Kriterien und Standards als Maßstäbe herangezogen werden oder für welche Nutzer(-gruppen) die Beurteilung vorgenommen wird. Die Qualitätsmessung gilt hiernach als eine notwendige Bedingung für das Abfassen eines Qualitätsurteils.

In einem praxisorientierten Verständnis steht Qualitätsmessung für jedwede Form der Untersuchung, mit der abgeklärt werden soll, ob und inwieweit die Fähigkeiten vorhanden sind, die festgelegten Qualitätsanforderungen zu erfüllen, aber auch, ob und inwieweit diese Anforderungen tatsächlich erfüllt wurden.

\subsubsection{Messtheoretische Grundlagen der Qualitätsmessung}

Qualitätsmessung oder allgemein die »Ermittlung von qualitätsrelevanten Daten « ist Grundlage jeder Auseinandersetzung mit dem Qualitätsbegriff, da sie über die Beschaffenheit wie auch über die Erfüllung, den Erfüllungsgrad oder aber die NichtErfüllung von Qualitätsanforderungen Auskunft geben kann. Über ihre Informations- und Darlegungsfunktion hinaus ermöglicht sie Veränderungen und zielgerichtetes Eingreifen in das Leistungs- und Versorgungsgeschehen. Bei extern veranlassten Qualitätsmessungen dominieren meist die Überwachungs- und Kontrollfunktion sowie die Funktion der Rechenschaftslegung (Legitimation). Die Aussagekraft der ermittelten Daten ist jedoch stets abhängig vom jeweiligen Erkenntnisinteresse und den methodischen Voraussetzungen (•Tab. 1.1).

Qualitätsmessung erfolgt in der Regel durch Qualitätskennzahlen oder -indikatoren. Ein Indikator ist eine Kenngröße, mit deren Hilfe unmittelbar nicht wahrnehmbare Zusammenhänge und Komplexe ausschnittsweise und stellvertretend abgebildet werden können. Es besteht eine »hypothetische Beziehung « zwischen dem gemessenen Indikator (z. B. Anzahl aufgetretener Stürze) und der abzubildenden Variable (z. B. Sicherheit als Kriterium der Pflegequalität), sodass ein indirektes Bild von der Qualität des jeweiligen Sachverhalts erzeugt wird. Auch wenn mithilfe von Qualitätskennzahlen 
Tab. 1.1 Qualitätsmessung im Gesundheitswesen:Verfahrenstechnische Ansätze und Anwendungsbereiche

Messansätze und -verfahren
(nach Schrappe 2017a, S. 8)

Quantitative Messungen: Erfassung und Analyse von

Daten definierter (unerwünschter) Ereignisse

Monitoring durch Indikatoren: Vorhersage von Problemen in der Gesundheitsversorgung

Analytisch generierende Verfahren: Identifizierung bisher noch unbekannter Faktoren

Komplexe Messungen zur wissenschaftlichen Evaluation: z. B. Veränderung klinisch-pflegerischer Outcomes (gesundheitliche Zustände, individuelles Verhalten, Lebensqualität etc.)
Haupteinsatz- und Anwendungsbereiche

(nach Geraedts 2009, S. 5)

Evaluation: Messung des Zielerreichungsgrads gegenüber vorgenannten Soll-Werten (Qualitätsbewertung)

Monitoring: Messung von Veränderungen beim Zielerreichungsgrad über die Zeit

Alarmfunktion: Messung von Situationen, die ein direktes Eingreifen erfordern (»Rote-Flagge«-Indikatoren).

Unterstützung externer Entscheidungsfindung: Wahlentscheidungen durch Nutzer, Zulassungsentscheidungen durch Behörden, Vergabe von Vergütungskomponenten etc. und Indikatoren einzelne Ereignisse und Merkmale des Leistungs- und Versorgungsgeschehens quantifiziert werden können, bleiben sie gewissermaßen nur Hilfsgrößen der Qualitätsbestimmung (JCAHO 1991; Idvall et al. 1997; Altenhofen et al. 2002).

Grundsätzlich setzt ein Indikator (oder eine Kennzahl) metrische Eigenschaften voraus und bildet in der Regel einen Zahlenwert (Mainz 2004). Im einfachsten Fall entspricht er einer einzigen Maßzahl (Grundzahl), die durch Zählen, Messen oder einer individuellen Einschätzung eines Sachverhalts ermittelt wird. Häufiger werden zwei Maßzahlen durch Quotientenbildung miteinander verknüpft und treten als Verhältniszahlen (z. B. Gliederungskennzahlen, Beziehungskennzahlen) in Erscheinung. Eine Indexzahl wiederum verknüpft rechnerisch einzelne Indikatorinformationen und fasst sie zu einer höher aggregierten Gesamtzahl zusammen (Bortz und Döring 2006, S. 143).

Indikatoren bilden in der Regel Teilaspekte des Leistungsgeschehens ab und ermöglichen Aussagen $\mathrm{zu}$ einem bestimmten Versorgungsausschnitt. Die Annäherung an ein komplexes Konstrukt (z. B. Pflegequalität einer Pflegeeinrichtung) im Sinne einer Gesamtbetrachtung ist allerdings nur durch die Verwendung mehrerer, nebeneinander zu betrachtender Indikatoren möglich (z. B. durch Indikatorprofile), die nur schwerlich in eine übergeordnete Maßzahl (z. B. aggregierte Indexzahl) zusammengeführt werden können. Je höher das gewählte Aggregations- und Abstraktionsniveau eines Indi- kators, desto hypothetischer bleibt die Beziehung zum abzubildenden Konstrukt.

Die Aussagekraft eines Indikators hängt von vielen methodischen Merkmalen ab. So ist für die Bewertung eines Indikators zunächst ein inhaltliches Verständnis für die Strukturen und Prozesse, die seine Ausprägung determinieren, notwendig (Kazandjian 1991). Als methodische Anforderungen gelten weiterhin: die Relevanz und der Nutzen für die Qualitätsverbesserung, die Wissenschaftlichkeit und Unterscheidungsfähigkeit des Indikators sowie die anwendungsorientierte Durchführbarkeit und Praktikabilität der Indikatorerhebung (Schyve 1995; Groene 2006; Reiter et al. 2008; Lüngen und Rath 2011, Schmitt et al. 2013). Die Konkretisierung dieser Anforderungen orientiert sich am jeweiligen Einsatzbereich und gewählten Messansatz. So sollten beispielsweise Indikatoren je nach Ansatz und Anwendungsbereich unterschiedlich in der Lage sein, entweder alle problematischen Fälle $\mathrm{zu}$ erfassen und falsch-negative Ergebnisse zu vermeiden (hohe Sensitivität), wie dies beim Monitoring-Ansatz verfolgt wird, oder "gute« von "schlechter « Qualität unterscheiden zu können und dabei falsch-positive Befunde vermeiden (hohe Spezifität), wie dies bei Evaluations-Ansätzen beabsichtigt ist (Schrappe 2017b). Bei linearen Messverfahren (quantitative Datenerfassung) bezieht sich die Validität auf die Erhebung selbst, d. h. ob das Messergebnis richtig wiedergegeben wird; bei sensitiv eingestellten Indikatoren mit Vorhersagefunk- 
tion auf die Vorhersage, also die Fähigkeit, diese Ereignisse mit der Qualität der Leistungserbringung in Zusammenhang zu bringen (Schrappe 2015).

\subsubsection{Modelltheoretische Vorbedingungen der Qualitätsbeurteilung}

Die einfachste Art der Qualitätsmessung ist das bloße Sammeln und Auflisten von Struktur- und Leistungsmerkmalen (z. B. Personalbestand, Anzahl versorgter Personen). Komplexer wird sie, wenn Merkmale der Prozess- und Ergebnisqualität ermittelt werden sollen (Nakrem et al. 2009). Allerdings kann allein mit der Darstellung von messbaren Ist-Zuständen kaum etwas über die tatsächliche (oder prognostizierte) Qualität ausgesagt werden.

Qualitätsmessung setzt idealerweise auf einem grundgelegten Qualitätsverständnis und einer akzeptierten Bewertungsgrundlage auf. Ausgangspunkt sind modellhafte Vorstellungen über die inhaltlichen Aspekte der darzustellenden Qualität sowie deren Ziele und Maßstäbe. Hochaggregierte Wertaussagen zur Versorgungsqualität (z. B. Effektivität, Angemessenheit) müssen zunächst für einen Versorgungsbereich (z. B. stationäre Langzeitpflege) definiert und nachfolgend auf einen relevanten Versorgungsaspekt (z. B. Unterstützung bei der Körperpflege, Arzneimittelgabe) heruntergebrochen werden (z. B. Richtigkeit der Ausführung von Tätigkeiten, Rechtzeitigkeit einer Pflegehandlung, Vollständigkeit der Dokumentation). Derart konkretisierte Wertaussagen zur Beurteilung der Qualität werden allgemein als Kriterien bezeichnet. Sie können normativ aus vorformulierten Qualitäts- und Praxisstandards (z. B. Expertenstandards), Qualitätsmodellen, Zertifizierungs- und Akkreditierungsstandards oder anderen vorgängigen Wissensquellen abgeleitet oder aber für einen bestimmten Einsatz- und Versorgungsbereich (professionsund organisationsbezogen) selbstständig entwickelt und festgelegt werden (Campbell et al. 2002; Altenhofen et al. 2002; Kötter et al. 2011; Büscher und Kabore 2014; Elsbernd 2014; Simon und Dunton 2014)

Der Begriff Kriterium wird uneinheitlich definiert und abstrahiert (Donabedian 1981). Im Kon- text der Qualitätsmessung kann er sowohl den Charakter einer übergeordneten Wertaussage annehmen als auch den einer formal umschriebenen Qualitätsanforderung, die sich auf messbare Merkmale des Leistungs- und Versorgungsgeschehens bezieht. Kriterien gelten daher auch als »Eigenschaften, deren Erfüllung typischerweise bei einer qualitativ hochwertigen Versorgung erwartet wird " (Geraedts et al. 2002). In einem enger gefassten Verständnis gehen mit dem Begriff Kriterium also nicht nur eine allgemeine Wertaussage, sondern auch ein konkreter Wertmaßstab und die Vorstellung von einem definierten Zielausmaß (Referenzgröße) des zu erfüllenden Qualitätsmerkmals einher ${ }^{4}$. Nur mithilfe eines festgelegten Zielausmaßes sind letztendlich Aussagen darüber möglich, ob und wann die Pflegehandlung oder die zu beurteilende Tätigkeit als »richtig «, »rechtzeitig « oder "vollständig" im Sinne einer qualitativ hochwertigen Versorgung bezeichnet werden kann.

Um die Ausprägung der hierfür zu messenden Merkmale bestimmen zu können, bedarf es der Auswahl eines geeigneten Dokumentations- und Messverfahrens (z. B. Pflegedokumentation, Zeitmessung, Zählkontrollen). Aus den hierüber erzeugten Daten werden Kenngrößen gebildet, die Auskunft über den gemessenen Ist-Zustand bezüglich eines Leistungs- und Versorgungsmerkmals liefern (deskriptiver Ansatz) oder in Gestalt eines Qualitätsindikators unter Hinzunahme eines Wertmaßstabs auch eine Aussage über die Erfüllung der an das Merkmal gestellten Anforderung ermöglichen (evaluativer Ansatz). Bei der praktischen Qualitätsmessung werden Einzelfallanalysen von kumulierten Analysen unterschieden, mit denen Aussagen über die individuelle (personen- bzw. fallbezogene) oder institutionelle (räumlich-zeitliche Datenaggregation) Versorgungs- oder Pflegequalität getroffen werden (• Abb. 1.2).

4 Inhaltlich vergleichbar und ähnlich variantenreich wird der Begriff "Standard" im Zusammenhang der Qualitätsmessung und -bewertung verwendet. Je nach Definition und Gebrauchsabsicht kann ein Standard den Charakter einer normativen Wertaussage annehmen, ein bestimmtes Leistungsniveau definieren oder eine präzise Nenngröße zur Spezifizierung eines adäquaten, akzeptablen oder optimalen Qualitätsniveaus liefern (Donabedian 1981; Gaebel und Schwarz 2006, S. 157; Brosnan 2012, S. 52). 


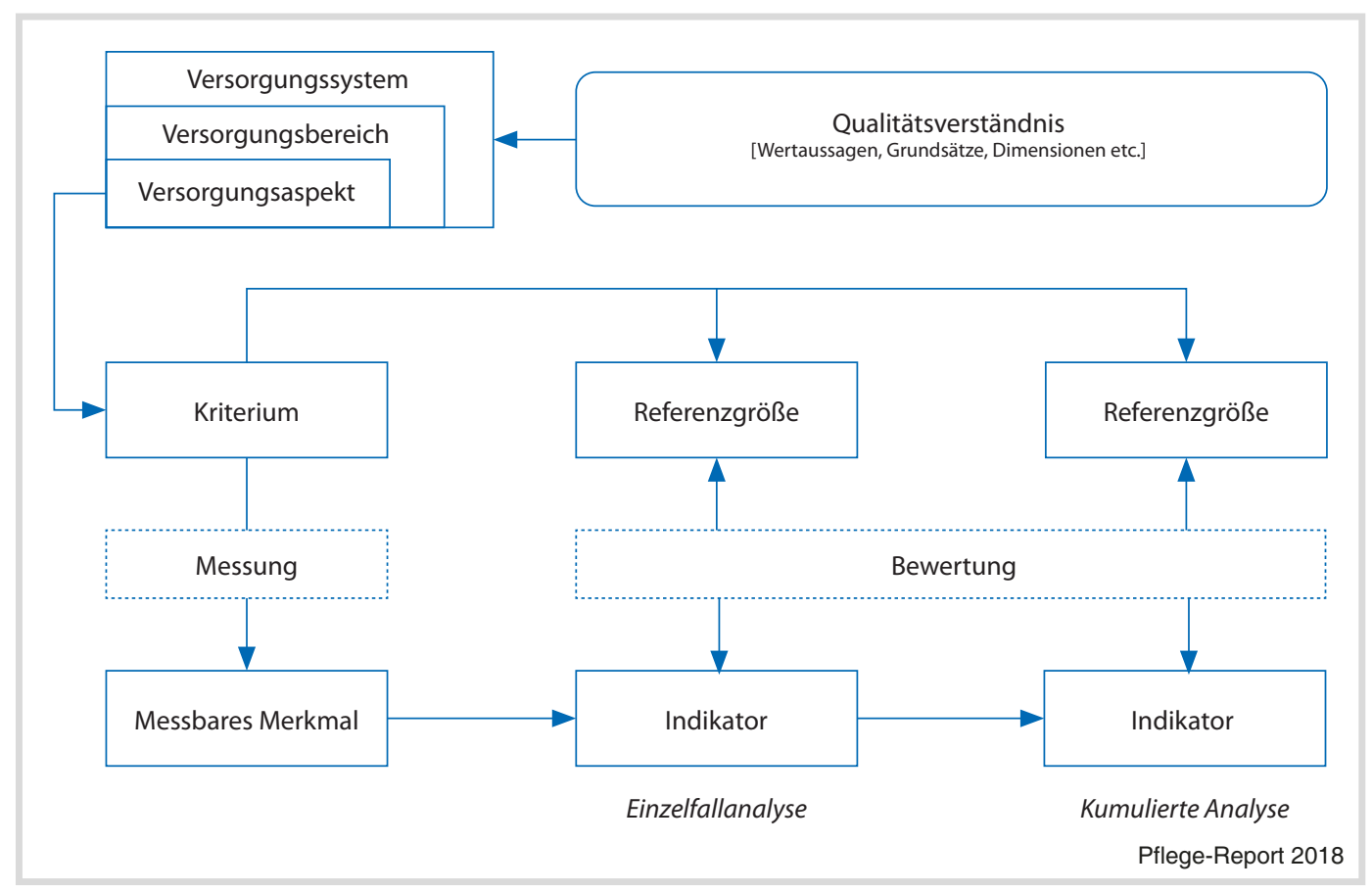

- Abb. 1.2 Messtheoretisches Grundmodell der Qualitätsmessung zur Unterscheidung von Einzelfallanalysen und kumulierten Analysen

Aussagen zur »tatsächlichen Qualität« bauen in der Regel auf diese Form der Datenerhebung auf. Dabei ist die Qualitätsbeurteilung methodisch stets an einen Vergleich mit den vorher definierten Zielen, Maßstäben und Referenzgrößen (Anforderungen) gekoppelt. In der Einzelfallanalyse wird zunächst die Ausprägung (Manifestation) des ausgewählten messbaren Merkmals ermittelt und mit der vorher festgelegten Referenzgröße verglichen (z. B. Zeitmessung innerhalb eines definierten Zeitfensters). Der Indikator der Einzelfallanalyse zeigt - als metrische oder kategoriale Kennzahl - an, ob oder in welchem Maße die umschrieben gestellte Anforderung (z. B. Rechtzeitigkeit der Probenentnahme) erfüllt ist, eine vorher definierte kritische Abweichung vorliegt (z. B. Überschreiten von Kontaminationsgrenzwerten) oder ein besonders folgenschweres Ereignis (z. B. unerwartete Todesfolge) eintrat. Kumulierte Analysen aggregieren Einzelfalldaten auf die Ebene definierter Leistungs- und Versorgungsbereiche (z. B. Abteilung, Gesamtorganisation). Als Indikatoren werden dann häufig
Gliederungszahlen gebildet, die als Rate das Auftreten bestimmter Ereignisse innerhalb eines bestimmten Zeitraums (z. B. Rate der Nichterfüllung, Komplikationsrate) oder als Quote den Anteil eines bestimmten Merkmals zu einem bestimmten Zeitpunkt (z. B. Fachkraftquote, Belegungsquote) abbilden.

\subsection{Zusammenfassende Schlussbetrachtung}

Pflegerische Versorgung ist weder umfänglich noch erschöpfend über messbare Kenngrößen erfassbar und darstellbar. In ihrer Grundausübung konstituiert sie sich vor allem durch ihre subjektive, einzelfallorientierte und situative Dimension und beinhaltet demgemäß auch Versorgungsaspekte, die sich "unmessbar" einer Quantifizierung und objektivierbaren Merkmalserhebung entziehen (z. B. Vertrauen, Wertschätzung, Zwischenmenschlichkeit). Andererseits ist Versorgungsqualität nicht allein 
durch die Beherrschung von Einzelhandlungen oder die Gestaltungsmacht und das Können einzelner Personen und Berufsgruppen erzielbar; sie ist auch an die Zweckrationalität und Funktionslogik ihres institutionellen Leistungsrahmens gebunden.

Qualität ist unabweislich ein multiperspektivischer Begriff, der unterschiedliche Zugänge und Deutungen zulässt; der aus einem institutionellen Gestaltungsanspruch sowie gesamtgesellschaftlichen Legitimationsverständnis zwangsläufig auch eine objektivierbare, überprüfbare und sichtbare Dimension fordert. Die Gestaltung, Steuerung und Entwicklung der Leistungen eines Versorgungsbereichs - individuell und institutionell - ist damit untrennbar an die Ermittlung, Darstellung und Beurteilung ihrer Qualität gekoppelt:

- Qualitätsmessung ist eine Querschnittsaufgabe: Sie macht Anforderungen transparent (»Soll«-Konzept), sie erhebt dazugehörige Merkmalsausprägungen und Sachverhalte des tatsächlichen Versorgungsgeschehens (»Ist «Konzept) und sie ermöglicht Aussagen über das Verhältnis von Anforderungs- und Leistungsebene (»Soll-Ist«-Konzept). Sie stellt die Grundlage für daten- und faktenbasiertes Handeln im Rahmen des versorgungsrelevanten Interaktionsgeschehens, des einrichtungsinternen Qualitätsmanagements, der externen Qualitätssicherung wie auch der qualitätsorientierten Versorgungssteuerung auf gesamtgesellschaftlicher Ebene.

- Qualitätsmessung ist Mittel, nicht Zweck: Sie orientiert sich an vorgängigen Annahmen und Aussagen über die Eigenschaften, die eine hochwertige Versorgung aufweisen sollte. Die notwendigerweise vorzunehmende Festlegung von Wertaussagen, Normen und Kriterien geht somit jeder Qualitätsmessung voraus; sie liefert Begründung wie auch Validierungsgrundlage für die Entwicklung und den Einsatz der erforderlichen Mess- und Erhebungsinstrumente, aber auch den erforderlichen Bewertungsrahmen für die Bildung eines Qualitätsurteils.

- Qualitätsmessung ist anspruchsvoll: Sie soll all jene Leistungs- und Versorgungsmerkmale sichtbar und bewertbar zu machen, die entlang eines bestimmten Qualitätsverständnisses als relevant und nutzenstiftend identifiziert werden. Hierzu ist der Messansatz dem jeweiligen Erkenntnisinteresse entsprechend auszuwählen und anzupassen. So unterliegen Indikatoren, die für Monitoring-Zwecke eingesetzt werden und auf Probleme im Versorgungsgeschehen hinweisen sollen, anderen methodischen Anforderungen als Indikatoren, die für eine retrospektive Qualitätsbewertung herangezogen werden.

In Zukunft wird die vergleichende Qualitätsmessung und -darstellung zunehmend mehr Daten und Informationen einfordern, mit denen das Leistungs- und Versorgungsgeschehen besser beurteilt, aber auch stärker kontrolliert und überwacht werden kann. Gleichzeitig wird Qualität bzw. die Darstellung von Qualität eine noch größere Rolle als bisher als Wettbewerbsfaktor für die Einrichtungen und Leistungserbringer spielen. Für beide Entwicklungen wird jedoch $\mathrm{zu}$ fordern sein, die Qualitätsmessung und -darstellung nicht nur wissenschaftlich ausreichend $\mathrm{zu}$ fundieren, sondern durch die Gesundheits- und Pflegeberufe stärker als bisher zu legitimieren.

Open Access Dieses Kapitel wird unter der Creative Commons Namensnennung 4.0 International Lizenz (http://creativecommons. org/licenses/by/4.0/deed.de) veröffentlicht, welche die Nutzung, Vervielfältigung, Bearbeitung, Verbreitung und Wiedergabe in jeglichem Medium und Format erlaubt, sofern Sie den/die ursprünglichen Autor(en) und die Quelle ordnungsgemäß nennen, einen Link zur Creative Commons Lizenz beifügen und angeben, ob Änderungen vorgenommen wurden.

Die in diesem Kapitel enthaltenen Bilder und sonstiges Drittmaterial unterliegen ebenfalls der genannten Creative Commons Lizenz, sofern sich aus der Abbildungslegende nichts anderes ergibt. Sofern das betreffende Material nicht unter der genannten Creative Commons Lizenz steht und die betreffende Handlung nicht nach gesetzlichen Vorschriften erlaubt ist, ist für die oben aufgeführten Weiterverwendungen des Materials die Einwilligung des jeweiligen Rechteinhabers einzuholen. 


\section{Literatur}

Allen-Duck A, Robinson JC, Stewart MW (2017) Healthcare Quality: A Concept Analysis. Nurs Forum 52 (4):377-386

Altenhofen L, Brech W, Brenner G, Geraedts M, Gramsch E, Kolkmann F-W, Krumpaszky HG, Lorenz W, Oesingmann $\mathrm{U}$, Ollenschläger G, Rheinberger P, Selbmann H-K, von Stillfried D, Strobawa F, Thole H (2002) Beurteilung klinischer Messgrößen des Qualitätsmanagements - Qualitätskriterien und -Indikatoren in der Gesundheitsversorgung. Konsenspapier von BÄK, KBV und AWMF. Z Arztl Fortbild Qualitatssich 96 (5):2-15

Arah OA, Westert GP, Hurst J, Klazinga NS (2006) A conceptual framework for the OECD health care quality indicators project. Int J Qual Health Care 18 (1):5-13

Attree M (1996) Towards a conceptual model of `Quality Care . Int J Nurs Stud 33 (1):13-28

Bortz J, Döring N (2006) Forschungsmethoden und Evaluation für Human- und Sozialwissenschaftler. Springer Medizin, Heidelberg

Brosnan CA (2012) Conceptual Models for Evaluation in Advanced Nursing Practice. In: Hickey JV, Brosnan CA (Ed.). Evaluation of Health Care Quality in Advanced Nursing Practice. Springer Publishing, New York, S 47-72

Büscher A, Kabore A (2014) Entwicklung von Qualitätsindikatoren auf der Basis von Expertenstandards. In: Schiemann D, Moers M, Büscher A (Hrsg) Qualitätsentwicklung in der Pflege. Konzepte, Methoden und Instrumente. Kohlhammer, Stuttgart, S 191-201

Campbell SM, Roland MO, Buetow SA (2000) Defining quality of care. Soc Sci Med 51 (11):1611-1625

Campbell SM, Braspenning J, Hutchinson A, Marshall M (2002) Research methods used in developing and applying quality indicators in primary care. Qual Saf Health Care 11 (4):358-364

Deming WE (1982) Out of the crisis. MIT Press, Cambridge, MA

Donabedian A (1966) Evaluating the Quality of Medical Care. Milbank Mem Fund Q 44 (3,Suppl):166-206

Donabedian A (1980) The Definition of Quality and Approaches to its Assessment. Explorations in Quality Assessment and Monitoring Volume 1. Health Administration Press, Ann Arbor, MI

Donabedian A (1981) Criteria, Norms and Standards of Quality: What Do They Mean? Am J Public Health 71 (4):409-412

Donabedian A (1990) The seven pillars of quality. Arch Pathol Lab Med 114 (11):1115-1118

Dotchin JA, Oakland JS (1992) Theories and concepts in total quality management. Total Quality Management 3 (2):33-46

Elsbernd A (2014) Entwicklung von Qualitätsindikatoren in der Altenpflege auf der Basis von Praxisstandards. In: Schiemann D, Moers M, Büscher A (Hrsg) Qualitätsentwicklung in der Pflege. Konzepte, Methoden und Instrumente. Kohlhammer, Stuttgart, S 202-215

Evans DB, Edejer TT, Lauer J, Frenk J, Murray CJ (2001) Measuring quality: from the system to the provider. Int J Qual Health Care 13 (6):439-446
Fließ S, Marra A, Reckenfelderbäumer M (2005) Betriebswirtschaftliche Aspekte des Pflegemanagements. In: Kerres A, Seeberger B (Hrsg) Gesamtlehrbuch Pflegemanagement. Springer, Berlin, S 396-436

Gaebel W, Schwarz M (2006) Qualitätsmanagement in der psychiatrischen Therapie. In: Möller H-J (Hrsg) Therapie psychischer Erkrankungen. Georg Thieme, Stuttgart, S 156-167

Geraedts M, Selbmann HK, Ollenschläger G (2002) Beurteilung der methodischen Qualität klinischer Messgrößen. Z Arztl Fortbild Qualitatssich 96:91-96

Geraedts M (2009) Einsatz von Qualitätsindikatoren. In: ÄZQ Ärztliches Zentrum für Qualität in der Medizin (Hrsg) Programm für Nationale Versorgungsleitlinien von BÄK, KBV und AWMF. Qualitätsindikatoren - Manual für Autoren. Verlag Make a Book, Neukirchen, S 5-7

Geraedts M, Holle B, Vollmar HC, Bartholomeyczik S (2011) Qualitätsmanagement in der ambulanten und stationären Pflege. Aktuelle Entwicklungen und Besonderheiten. Bundesgesundheitsblatt Gesundheitsforschung Gesundheitsschutz 54 (2):185-193

Groene O (2006) Vorschläge der WHO zur umfassenden Leistungsbewertung von Krankenhäusern. Gesundh ökon Qual manag 11:226-233

Harteloh PPM (2003) The meaning of quality in health care: A concept analysis. Health Care Anal 11 (3):259-267

Hensen P (2016) Qualitätsmanagement im Gesundheitswesen. Grundlagen für Studium und Praxis. Springer Gabler, Wiesbaden

Hensen P (2018) Qualitätsentwicklung zwischen Institution und Interaktion - Eine Standortbestimmung aus professionstheoretischer Sicht. In: Hensen P, Stamer M (Hrsg) Professionsbezogene Qualitätsentwicklung im interdisziplinären Gesundheitswesen. Springer VS, Wiesbaden, S 3-67

Idvall E, Rooke L, Hamrin E (1997) Quality indicators in clinical nursing: a review of the literature. J Adv Nurs 25 (1): 6-17

Institute of Medicine (IOM) (1990) Medicare. A Strategy for Quality Assurance, Volume II: Sources and Methods. The National Academies Press, Washington, DC

Institute of Medicine (IOM) (2001) Crossing the quality chasm: A new health system for the 21 st century. The National Academies Press, Washington, DC

Joint Commission on Accreditation of Healthcare Organisations (JCAHO) (1991) Primer on indicator development and application. Measuring quality in health care. Joint Commission on Accreditation of Healthcare Organisations, Oakbrook Terrace, IL

Kazandjian VA (1991) Performance indicators: pointer dogs in disguise - a commentary. J Am Med Rec Assoc 62 (9):34-36

Kleinaltenkamp M (2001) Begriffsabgrenzung und Erscheinungsformen von Dienstleistungen. In: Bruhn M, Meffert H (Hrsg) Handbuch Dienstleistungsmanagement. Gabler, Wiesbaden, S 27-50

Kötter T, Schaefer F, Blozik E, Scherer M (2011) Die Entwicklung von Qualitätsindikatoren - Hintergrund, Methoden 
und Probleme. Z Evid Fortbild Qual Gesundh wesen 105 (1):7-12

Lüngen M, Rath T (2011) Analyse und Evaluierung des QUALIFY Instruments zur Bewertung von Qualitätsindikatoren anhand eines strukturierten qualitativen Interviews. Z Evid Fortbild Qual Gesundh wesen 105 (1):38-43

Mainz J (2004) Quality indicators: essential for quality improvement. Int J Qual Health Care 16 (Suppl 1):i1-i2

Maxwell RJ (1992) Dimensions of quality revisited: from thought to action. Qual Health Care 1 (3):171-177

Merchel J (2013) Qualitätsmanagement in der Sozialen Arbeit. Eine Einführung. Beltz Juventa, Weinheim Basel

Meyer A, Mattmüller R (1987) Qualität von Dienstleistungen. Entwurf eines praxisorientierten Qualitätsmodells. Marketing ZFP 9 (3):187-195

Nakrem S, Vinsnes AG, Harkless GE, Paulsen B, Seim A (2009) Nursing sensitive quality indicators for nursing home care: international review of literature, policy and practice. Int J Nurs Stud 46 (6):848-857

$\varnothing$ vretveit J (1992) Health service quality. Blackwell Scientific Press, Oxford

Piligrimiene K, Buciuniene Z (2008) Different Perspectives on health care quality: is the consensus possible? Engineering Economics 1 (56):104-110

Prütz F (2012) Was ist Qualität im Gesundheitswesen? Ethik Med 24:105-115

Rantz MJ, Mehr DR, Popejoy L, Zwygart-Stauffacher M, Hicks LL, Grando V, Conn VS, Porter R, Scott J, Maas M (1998) Nursing home care quality: a multidimensional theoretical model. J Nurs Care Qual 12 (3):30-46

Rantz MJ, Zwygart-Stauffacher M, Popejoy L, Grando VT, Mehr DR, Hicks LL, Conn VS, Wipke-Tevis D, Porter R, Bostick J, Maas M, Scott J (1999) Nursing home care quality: a multidimensional theoretical model integrating the views of consumers and providers. J Nurs Care Qual $14(1): 16-37$

Reerink E (1990) Defining quality of care: mission impossible? Qual Assur Health Care 2 (3-4):197-202

Reiter A, Fischer B, Kötting J, Geraedts M, Jäckel WH, Döbler K (2008) QUALIFY: Ein Instrument zur Bewertung von Qualitätsindikatoren. Z Arztl Fortbild Qualitatssich 101: 683-688

Ruckstuhl B, Kolip P, Gutzwiller F (2001) Qualitätsparameter in der Prävention. In: Bundeszentrale für gesundheitliche Aufklärung (Hrsg) Qualitätsmanagement in Gesundheitsförderung und Prävention. Grundsätze, Methoden und Anforderungen. BzgA, Köln, S 38-50

Schmitt J, Petzold T, Eberlein-Gonska M, Neugebauer EA (2013) Anforderungsprofil an Qualitätsindikatoren. Relevanz aktueller Entwicklungen der Outcomes Forschung für das Qualitätsmanagement. Z Evid Fortbild Qual Gesundh wesen 107 (8):516-522

Schrappe M (2015) Aktuelle Ansätze zur Qualitätsverbesserung in der Gesundheitsversorgung - eine kritische Würdigung. GGW 15 (3):7-14
Schrappe M (2017a) Pay for Performance - Aktueller Stand und Perspektiven. In: Dormann F, Klauber J (Hrsg) Qualitätsmonitor 2017. Medizinisch Wissenschaftliche Verlagsgesellschaft, Berlin, S 3-14

Schrappe M (2017b) Das Methodenpapier des IQTIG: keine Kursänderung in Sicht. Die ex post-Qualitätskontrolle bleibt die vorherrschende Doktrin. Monitor Versorgungsforschung 10 (2):41-45

Schyve P (1995) Models for relating performance measurement and accreditation. Int J Health Plann Manage 10:231-241

Simon M, Dunton N (2014) Entwicklung, Erprobung und Anwendung von Qualitätsindikatoren der Pflege im Krankenhaus: das Beispiel NDNQI ${ }^{\circledR}$ aus den USA. In: Schiemann D, Moers M, Büscher A (Hrsg) Qualitätsentwicklung in der Pflege. Konzepte, Methoden und Instrumente. Kohlhammer, Stuttgart, S 216-222

Verbeck A (1998) TQM versus QM. Wie Unternehmen sich richtig entscheiden. vdf Hochschulverlag, Zürich

Zink, KJ, Schildknecht R (1994) Total Quality Konzepte Entwicklungslinien und Überblick. In: Zink KJ (Hrsg) Qualität als Managementaufgabe. Mi-Wirtschaftsbuch, München, S 73-108 